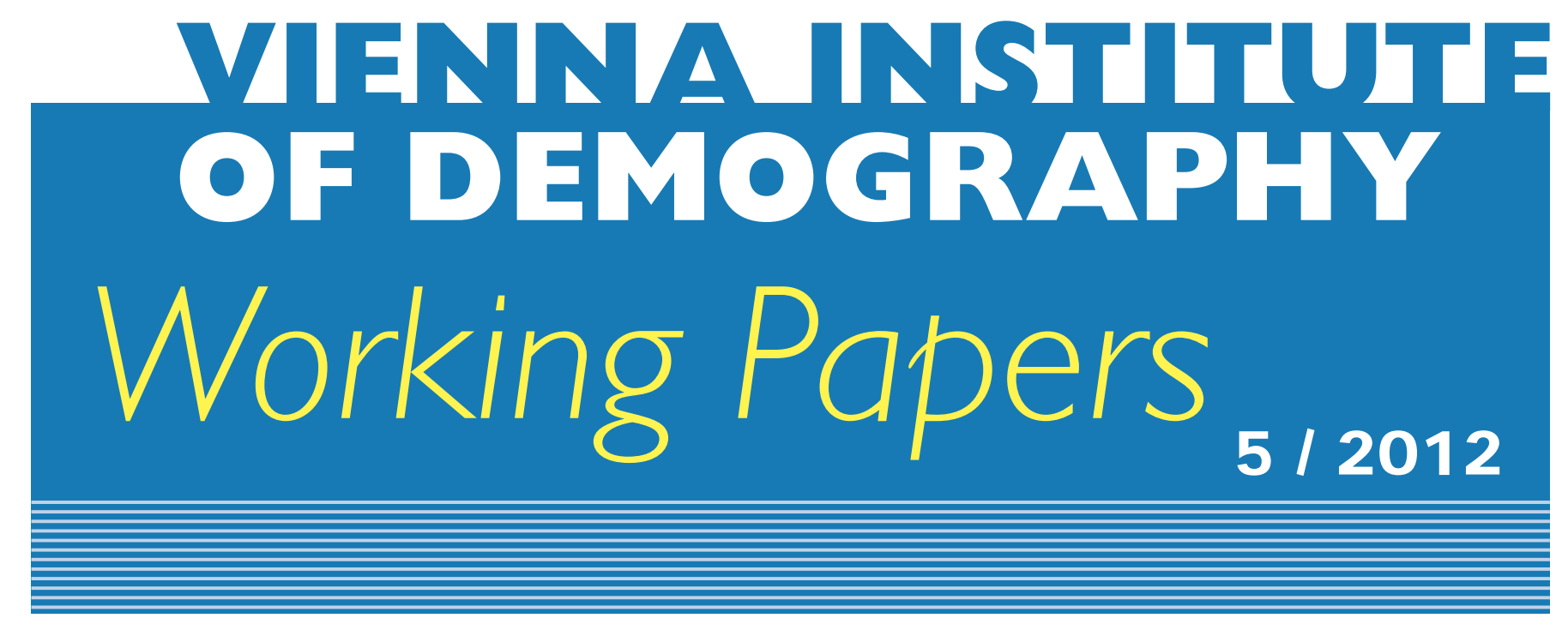

Nikola Sander and Martin Bell

\title{
Age, Period and Cohort Effects on Migration of the Baby Boomers in Australia
}

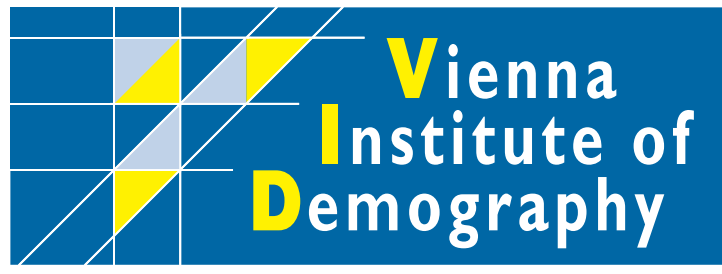

Vienna Institute of Demography Austrian Academy of Sciences

Wohllebengasse 12-14

A-I040Vienna $\cdot$ Austria

E-Mail:vid@oeaw.ac.at

Website: www.oeaw.ac.at/vid 


\begin{abstract}
The differential migration behaviour of the baby boom generation is commonly explained by reference to cohort size effects. This paper focuses on inter-cohort differences in the intensity and pattern of internal migration in Australia, while paying particular attention to the Australian baby boomer generation as it has moved through the life-course. A series of generalised linear models are fitted to migration transition for age-period-cohort spaces to disentangle the effects of age, period and birth cohort on migration. The results demonstrate that the relative contribution of age to change in migration intensity is largest in relative terms, followed by cohort, while the effects of period were much more subtle. In comparison to earlier work on cohort effects in the United States that showed a negative effect of large cohort size on migration, cohort effects on migration are not restricted to the baby boom but show a continuous upwards trend across cohorts. We find that this divergence from the US-based evidence regarding cohort effects is largely due to the different timing and magnitude of the Australian baby boom compared to the US. The baby boom in Australia was delayed and much smaller in terms of absolute and relative cohort size than in the US. Results highlight the importance of factors other than cohort size, such as differences in attitudes to mobility and housing market dynamics, in shaping migration behaviour of the Australian baby boomers.
\end{abstract}

\title{
Keywords
}

Internal migration, cohort analysis, baby boom, Australia

\section{Authors}

Nikola Sander is a post-doctoral research scholar at the Wittgenstein Centre (IIASA, VID/ÖAW, WU), Vienna Institute of Demography/Austrian Academy of Sciences. Email: Nikola.Sander@oeaw.ac.at

Martin Bell is a Professor for Human Geography and Director of the Queensland Centre for Population Research at The University of Queensland, Australia. Email: martin.bell@uq.edu.au

\section{Acknowledgements}

The work presented here forms part of doctoral research undertaken by the first author and funded by the University of Queensland under an International Research Award from 2006 to 2010. This paper was supported financially by an Australian Research Council Discovery Grant (DP 0451399). The authors would like to thank Warren Sanderson, Vegard Skirbekk and Dimiter Philipov for their helpful comments on an earlier version of the paper. 


\title{
Age, Period and Cohort Effects on Migration of the Baby Boomers in Australia
}

\author{
Nikola Sander and Martin Bell
}

\section{Introduction}

The oldest members of the baby boomer generation will soon reach retirement age. The unprecedented rise in people withdrawing from the labour force will not only have striking fiscal implications, but will also result in an increased pool of potential retirement migrants. Even if retirement migration intensities stay constant, the size of cohorts will lead to an increased volume of retirement migration. However, the large size of the baby boomer generation, altered fiscal circumstances of retirees, as well as fluctuations in the housing and labour markets may cause migration intensities to change in the future. To understand how these interrelated factors have shaped migration behaviour as the baby boomers have moved through the life-course so far, the effects of age, period and cohort have to be disentangled using age-period-cohort (APC) models. However, the application of APC models to internal migration has been hindered by the lack of data that are classified simultaneously by age, period and birth cohort (Bell and Rees, 2006).

The limited empirical evidence on migration of the baby boomer generation is equivocal and dominated by US studies (Frey, 1986; Haas and Serow, 2002; Pandit, 1997a; Plane, 1992; Plane and Rogerson, 1991). Focusing on the intensity of migration among the young adult baby boomers as they entered the labour market during the 1970s and $1980 \mathrm{~s}$, mobility rates were shown to be reduced among members of large cohorts (Pandit, 1997a; Pandit, 1997b; Plane and Rogerson, 1991; Rogerson, 1987). The decline in migration rates during the time of entry into the labour force was attributed to increased competition for jobs among members of large cohorts (Plane and Rogerson, 1991). Pandit (1997a) showed that it is the joint effect of cohort size and economic conditions which shapes migration intensities, and that the effects were more pronounced for longer-distance moves than for short-distance moves. Pandit also argued that cohort size effects were more important in relative terms, which means that the positive effect of economic growth and low unemployment is more than offset by the negative effect of large cohort size.

The aim of this paper is to determine how cohort size and economic conditions have shaped the migration dynamics of the Australian baby boomers as they moved through the life-course, and to elaborate on the US findings in the Australian context. This study overcomes the problem of data sparseness by using migrant counts estimated for ageperiod-cohort (APC) spaces (Bell and Rees, 2006). Migrant counts estimated for APC spaces are used to break the relationship of period $p$ - age $a=$ cohort $c$, which avoids the identification problem in age-period-cohort models. Thus, the approach adopted here represents an alternative to the extensively discussed constrained generalized linear model (Fienberg and Mason, 1979; Mason and Smith, 1985) and the intrinsic estimator method 
(Yang et al., 2008). The results are compared to those reported by Pandit (1997a; 1997b) and Plane and Rogerson (1991) for the US.

This paper begins with a discussion of how age, period and cohort effects can be disentangled, and then turns to consider the size and timing of the Australian baby boom cohort, and changes over time in the labour and housing markets. Next, the paper introduces the data, the spatial framework and the APC models. The results section commences with an analysis of the model fit, and then proceeds to evaluate separately the effects of age, period and cohort on migration. The paper then discusses the effects of cohort size and economic conditions, followed by the identification of differences in APC effects by type of move.

\section{Disentangling Age, Period and Cohort Effects}

Although APC models have been widely used in the study of demographic epidemiological and social trends (O'Brien, 2000; Robertson and Boyle, 1998; Wilmoth, 1990), estimating the parameters for age, period and cohort is not straightforward. Using linear regression of migrant counts classified by period and cohort yields the problem of a linear dependency between age, period and birth cohort (Mason et al., 1973). When migration data for period-cohort spaces are collected for five-year transitions and by fiveyear age groups, the linear dependency implies that: if a count of migrants in the ten to 14 age group was collected in the census period 1981-86, one can determine that these migrants (a) were aged five to nine years at the beginning of the transition interval, and (b) were born in 1971-76. The identifiability problem in APC analysis (i.e. the relationship of Period - Age $=$ Cohort) has attracted scholarly attention among epidemiologists and sociologists for several decades but has not yet been fully resolved (Mason and Fienberg, 1985; Mason and Smith, 1985; Yang, 2007; Yang et al., 2008).

The identifiability problem can be eliminated if migrant counts estimated for APC spaces are used in a generalised linear modelling approach. Disaggregating period-cohort transitions into APC spaces avoids the identifiability problem (Bell and Rees, 2006). Revisiting the example of migrant counts for the ten to 14 age group collected in 1981-86 discussed earlier, splitting the period-cohort space in two APC spaces means that the migrant count collected for the 1971-76 birth cohort in the period 1981-86 is now segmented into those migrants aged five to nine years and those aged ten to 14 years at the time of the move, thus breaking the linear dependency.

\section{The Australian Baby Boom}

The literature in the US suggests that large cohort size had a depressing effect on migration intensities (Haas and Serow, 2002; Pandit, 1997a; Plane and Rogerson, 1991). However, the effect of birth cohort size on migration intensities does not necessarily hold in the Australian context. This is because the Australian baby boom is different in timing and magnitude from that in the US. Based on annual births, the baby boom in Australia was delayed and, even more importantly, much smaller in terms of cohort size than in the 
US. Figure 1 shows the number of live births for Australia (in 100,000) and the US (in millions) for the period 1948 to 1990. As in the US, the increase in live births in Australia was partly caused by a catch-up effect associated with births postponed during the Great Depression and World War II. However, the Australian baby boom occurred approximately ten years later and the increase in the number of births between 1946 and 1961 was much lower. The drop in the number of births between 1961 and 1965 was due to delays in child bearing associated with increased education and workforce participation by women, and the introduction of the oral conceptive pill in 1961 (ABS, 1998). In 1966, it was the start of a marriage boom that resulted in a reduction in the average age at first marriage of women and an increase in the number of births, which peaked sharply in 1971. As a result, US birth cohorts born in the late 1950s and early 1960s are larger than those born in the 1980s and 1990s, while in Australia, the size of the cohorts born in the 1980s and 1990s is larger than those born in the 1960s. However, Australians born after 1986 represent the baby boom echo and are even larger than the baby boom.

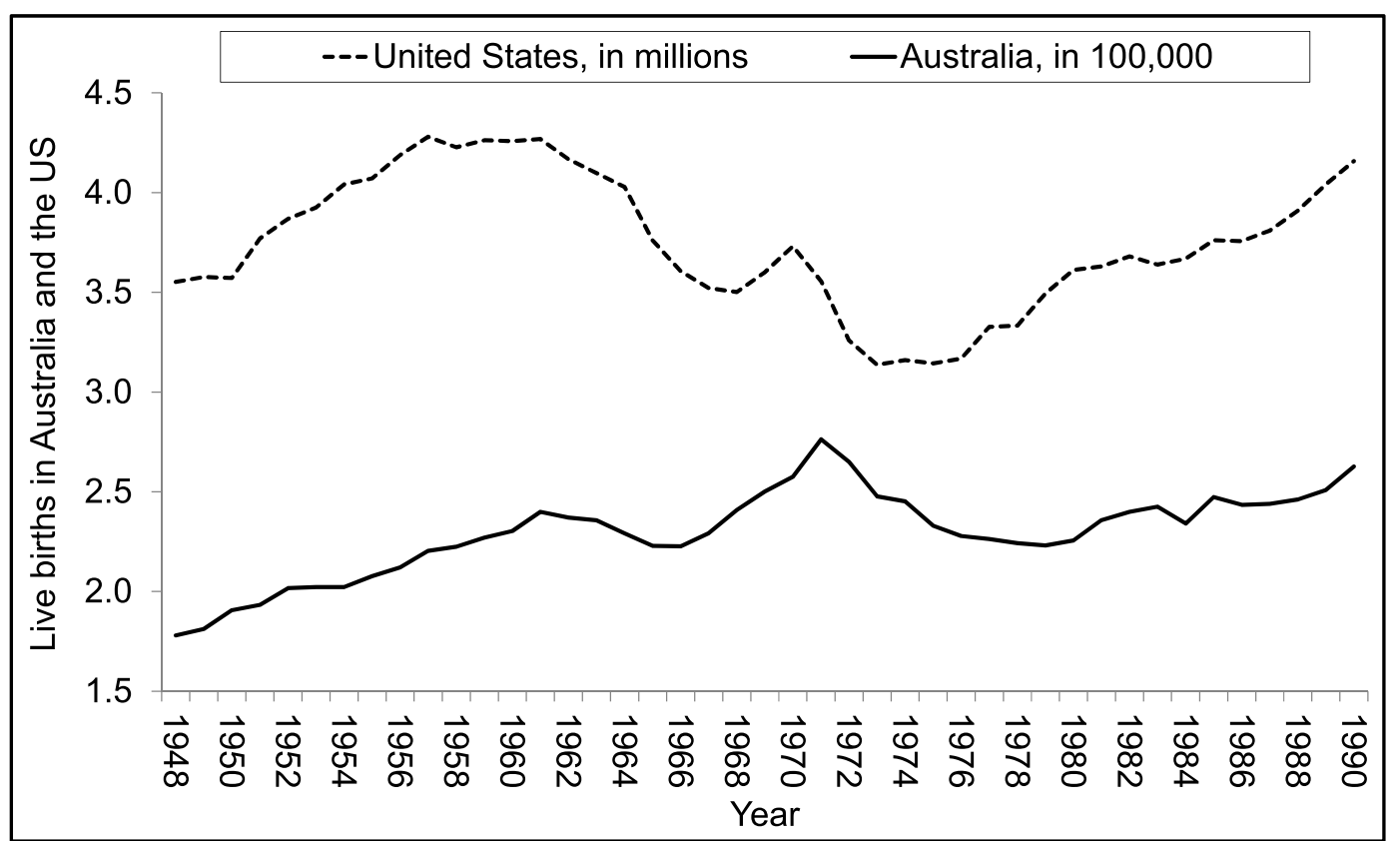

Data sources: Australian Bureau of Statistics, United Nations and National Centre for Health Statistics

Figure 1 Live births in Australia and the US, 1948-1990

The literature suggests that large relative cohort size at entry into the labour and housing markets discourages mobility (Pandit, 1997a; Plane and Rogerson, 1991). Changes over time in the annual number of births implicitly affect the relative size of birth cohorts as they move through the life-course. Since the peak in the number of births occurred later in Australia and was less pronounced than in the US, the demographic bulge created by the baby boom was much smaller in Australia. When the boomers entered the labour and housing markets in the late 1970s and early 1980s, the larger bulge of US boomers meant that the percentage of 15-24 year olds in the population was much larger in the US (see Figure 2). In 1955, when the small pre-baby boom cohorts entered the labour force, the proportion of 15-24 year olds was 13 per cent in both countries. By 1980, the proportion had increased to 18.6 per cent in the US, whereas in Australia the rise was much slower 
(17.5 per cent). The higher proportion of young adults in the Australian population in the 1990s reflects the later peak in the number of births compared to the US. The smaller bulge of Australian baby boomers means that there may have been less competition as they entered the labour and housing markets.

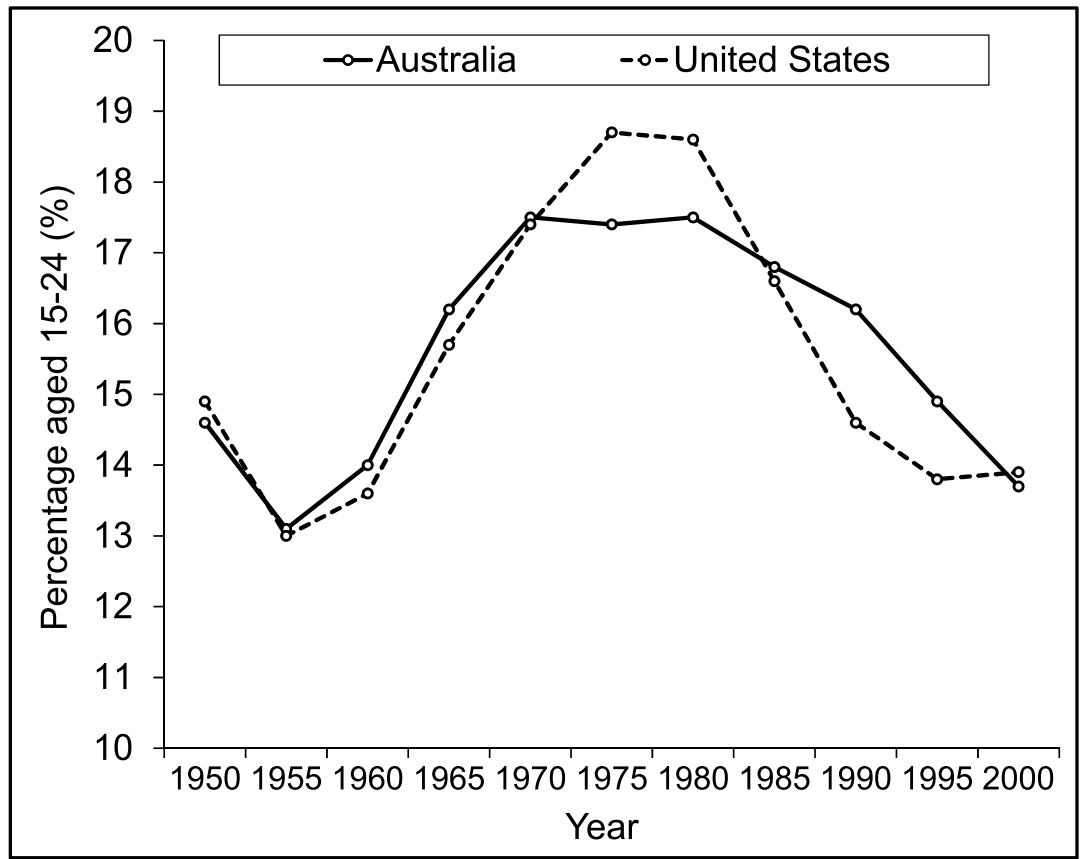

Data source: UN Population Division World Population Prospects: The 2008 Revision

Figure 2 Percentage of the population aged 15-25 years in Australia and the US, 1950-2000

\section{Labour and Housing Market Change}

As the baby boomers moved through the life-course, they encountered a sequence of economic events. Figure 3 shows the housing interest rate, the unemployment rate for young adults aged 20-29 years and the real GDP per capital for the period that census data on migration are available for, 1976 to 2001. Australia experienced strong economic growth and low unemployment in the early 1970s. The international oil crisis, restructuring in some industry sectors and weakening economic growth resulted in increasing unemployment and high interest rates by the early 1980s. In 1983, the unemployment rate peaked at above ten per cent. The housing market boom in the 1980s was paralleled by very high interest rates above 15 per cent from 1986 to 1990 , undoubtedly reducing housing affordability. The recession of the Australian economy and the marked decline in manufacturing employment in the early 1990s resulted in a renewed increase in unemployment rates to above ten per cent in 1992 and 1993, while housing affordability increased in the 1990s. The restructuring of the manufacturing sector and the economic recession during the early 1990s meant that GDP per capita grew more slowly during that time, although the general trend in GDP was clearly upwards. 


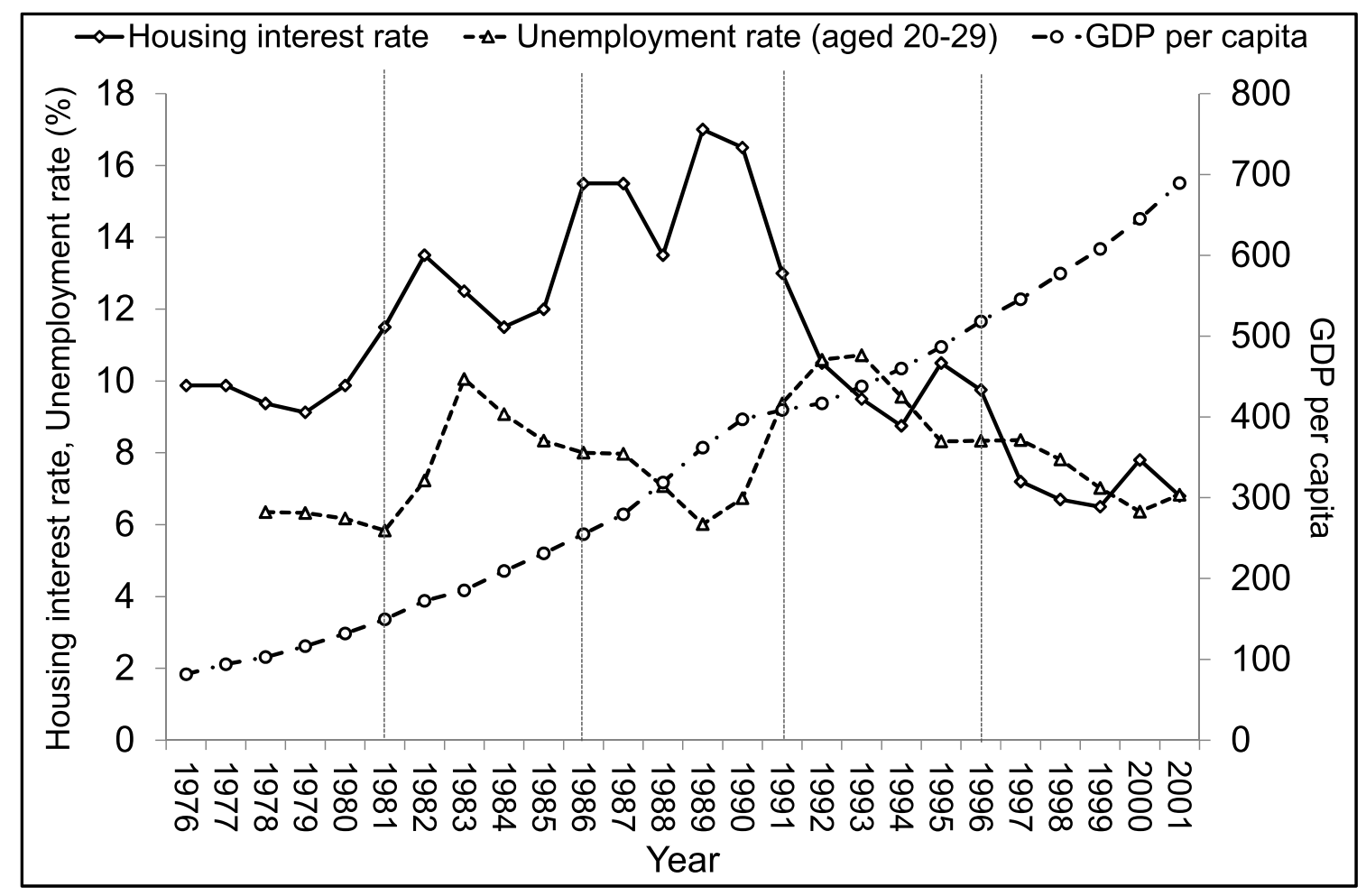

Figure 3 Changes in housing interest rates, unemployment rates and GDP per capita, 1976 to 2001, vertical lines indicate quinquennial census periods

The North American literature demonstrated that the propensity to move was lower during periods of economic recession and high unemployment in the 1970s and 1980s (Milne, 1993). The effects of these structural forces on migration were most pronounced among young-adult baby boomers as they entered the US labour and housing markets (Haas and Serow, 2002; Pandit, 1997a; Plane and Rogerson, 1991). However, making the distinction between period and cohort effects is not straightforward. Period effects arise if economic depression or real estate booms affect the migration intensity among the whole population. A cohort effect occurs if the depression only affects mobility levels among a certain age group, which is a particular birth cohort (Blossfeld, 1986). To elaborate on the US findings in the Australian context, the impact of economic indicators (i.e. GDP, housing interest rate and unemployment rate of 20-29 year olds) on migration was determined in the analysis presented here.

To further complicate the cross-national comparison, economic conditions that prevailed in Australia as the baby boomers entered the labour and housing markets were very different from those in the US. In Australia, people enter the labour force aged 17 to 24 years and first home buyers are in their late 20s and early 30s (Flatau et al., 2003; Rodrigues, 2003). This means that the older baby boomers born 1946 to 1976 entered the labour market during times of economic growth and low unemployment (1960s and 1970s), and purchased their first home from the 1970s to early 1990s. In contrast, the younger baby boomers born 1961 to 1976 entered the labour market during a period of high unemployment in the early 1980 s, and they were in the housing market entry stage during the interest rate peak in the late 1980s. 
In considering these differences in the economic conditions and the magnitude of the baby boom between the two countries, four key questions emerge that will help determine whether the findings reported by Pandit (1997a; 1997b) and Plane and Rogerson (1991) hold in the Australian context: Has the intensity of migration been lower among members of the baby boom generation compared to earlier and later cohorts? Is the intensity of migration negatively correlated with birth cohort size? Have cohort effects been more influential in shaping migration behaviour than economic conditions or housing cost? Do cohort effects differ by type of move?

\section{Data}

The analysis presented in this paper uses five-year migration transition data from the Australian Internal Migration (AIM) database, database, disaggregated by sex, fiveyear age groups and five-year birth cohorts for the five quinquennial census periods 197681 to 1996-2001. The period-cohort data reported in the census capture migrants that belong to a given birth cohort in a given five-year period. Age is not measured at the time of the move but at the end of the five-year period. Thus, a given cohort is aged five years younger at the beginning of the period. For a complete description of the AIM database, the reader is referred to Bell et al. (1999) and Rees et al. (2000)

Migrant counts for APC spaces were calculated by splitting the period-cohort spaces shown in Figure 4 into two APC spaces, a younger and an older APC space. Since mobility varies strongly by age, it is not feasible to simply divide the period-cohort spaces into equal parts. Instead, a set of separation factors was used. These factors were derived from single-year national mobility profiles for one-year age groups as provided in Bell and Rees (2006). The separation factors represent the proportion of mobility in a given APC space of the total mobility in the corresponding period-cohort space. Following the approach adopted by Bell and Rees (2006), for each period, the period-cohort counts (originally disaggregated into 15 age groups) were split into 29 APC spaces according to the following equation (see Figure 4).

$$
\mathrm{APC}_{\mathrm{ij}(\text { young) }}=\mathrm{PC}_{\mathrm{ij}} * \mathrm{SF}_{\mathrm{ij}}
$$

where $A P C_{i j(y o u n g)}$ is the younger APC space in period $i$ at age $j$ (APC 2 in Figure 4), $P C_{i j}$ is the period-cohort migrant count in period $i$ at age $j$ and $S F_{i j}$ is the separation factor for the period-cohort count in period $i$ and at age $j$. Note that no older APC space is calculated for the $75+$ age group. The final dataset thus comprises 145 observations (29 APC spaces over five periods) for each origin-destination flow. To relate the migrant counts to the size of the population in the origin region, the counts are divided by the PAR of migrating. The PAR was identical for the younger and the older APC space in period $i$ at age $j$. PAR was defined as the number of persons at age $j$ living in the origin at the beginning of the period $i$ (Bell and Rees, 2006). Since the data are collected over the 25year period 1976-81 to 1996-2001, they do not cover the entire life-course of cohorts. Instead, they provide a snapshot of each cohort's mobility. For example, migrant counts for the cohort born 1921-26 are collected for the age groups of 55-59, 60-64, 65-69, 70-74 and $75+$ years. 


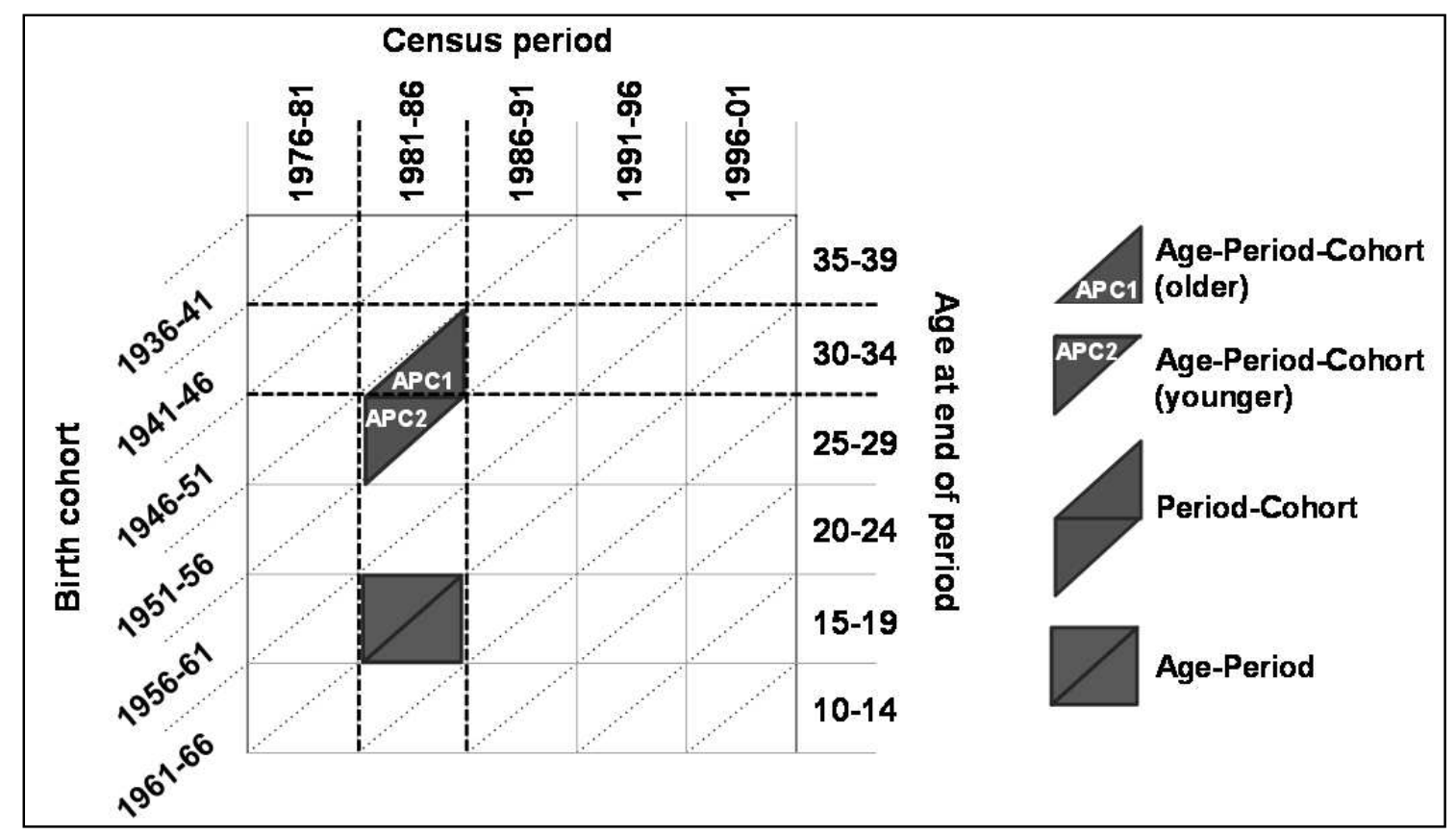

Figure 4 Migration data observation plans

Data on birth cohort size were obtained from the Australian Bureau of Statistics (ABS). To evaluate the impact of cohort size on migration, cohort size records were divided into four quartiles (four classes with approximately equal membership counts). These quartiles were then used to group cohorts into three groups: those cohorts with a size below the 0.25 quartile ( 25 per cent of the cohort size values fall below the 0.25 quartile) are classed as 'small', cohorts with a size above the 0.25 quartile and below the 0.75 quartile are classed as 'medium', and cohorts with a size above the 0.75 quartile are classed as being 'large' in size. This means that there are 621,000 to 796,000 live births in the small five-year birth cohorts, 796,001 to $1,360,000$ live births in the medium size cohorts and $1,360,001$ to $1,550,000$ live births in the large cohorts.

Data on GDP and unemployment rates of 20-29 year olds for the period 1976-2001 were obtained from the ABS to determine the effects of economic circumstances, housing market conditions and labour market processes on migration intensity in an Australian context. Data on housing interest rates (per cent per annum) were obtained from the Reserve Bank of Australia.

\section{Spatial Framework}

Changes to administrative boundaries used for census data collection represent a commonly encountered problem in the time-series analysis of migration. Hence, comparisons of migration behaviour across periods and cohorts cannot be made unless the boundary changes are adjusted for. The AIM database holds information on migrant flows 
between 69 so-called Temporal Statistical Divisions (TSDs) with consistent boundaries. The TSDs provide a detailed regional breakdown of Australia and cover the whole continent. The capital cities in New South Wales, Victoria, Queensland, South Australia and Western Australia are each represented by three TSDs (inner, middle and outer), the less populous capital cities in Tasmania, the Northern Territory and the Australian Capital Territory are represented by a single TSD, and the non-metropolitan parts of the six states are split into a variable number of regions, ranging from 12 in Queensland to three in Tasmania.

In light of the findings presented by Plane (1992) and Pandit (1997a) on the distinctive pattern of migration among young-adult baby boomers in the US, the primary interest here was to investigate variations in the relativities of period and cohort effects by type of move. The settlement pattern of Australia is unique in that two-thirds of the total population live in the capital cities of the seven states and territories. All capital cities except Canberra are located on the coastline. In contrast, most of the interior is very sparsely populated with a population density of less than five persons per square kilometre. Given the high urban primacy in the Australian settlement pattern, a simple dichotomy was used to classify regions into metropolitan and non-metropolitan (see Figure 5).

Specifically, metropolitan TSDs in the capital cities of Sydney, Melbourne, Brisbane, Adelaide and Perth were aggregated to form single regions, while nonmetropolitan TSDs were not aggregated. Separate APC models were run for each of the following five types of movements:

- within the same TSD (i.e. within a metropolitan or a non-metropolitan TSD);

- from a metropolitan to a non-metropolitan TSD in the same state;

- from a non-metropolitan to a metropolitan TSD in the same state;

- from a non-metropolitan to another non-metropolitan TSD in the same state; and

- interstate (i.e. to a metropolitan or non-metropolitan TSD in a different state) 


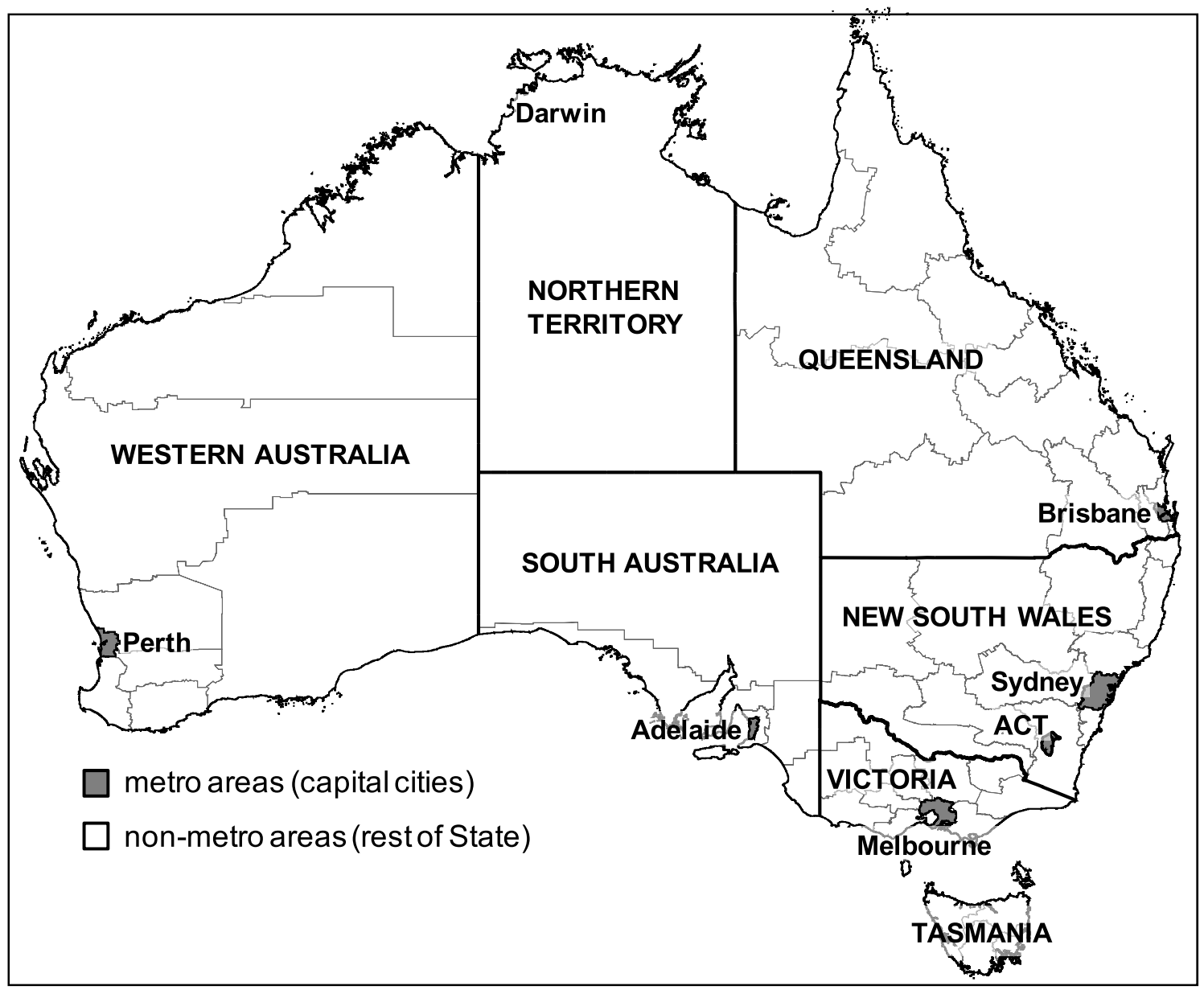

Figure 5 Classification of TSDs as metropolitan and non-metropolitan

\section{Age-Period-Cohort Models}

The analytical approach used here aims to identify how the effects of age, time period and birth cohort have influenced the aggregate intensity of migration over the last 30 years. While the focus of the analysis is on the baby boomers, the effects of age, period and cohort are determined initially for the whole population. Whether the baby boomers migrated more or less than preceding and succeeding cohorts will then be assessed by comparing the baby boom cohort (born 1946-51 to 1971-76) to the pre-baby boom (born 1901-06 to 1941-46) and the post-baby boom generations (born 1976-81 to 1991-96). These three cohorts each consist of people born in the same period, and as people age, they pass through a sequence of life-course stages. Each cohort passes through these stages at different times and in different historical contexts. Figure 6 shows a Lexis diagram that plots age on the vertical axis against time on the horizontal axis and cohort on the diagonal. The diagram depicts the movement of the pre-baby boomers, the boomers and the postbaby boomers through a series of life-course stages as the cohorts entered the labour market, the housing market and retirement at different historical times. 


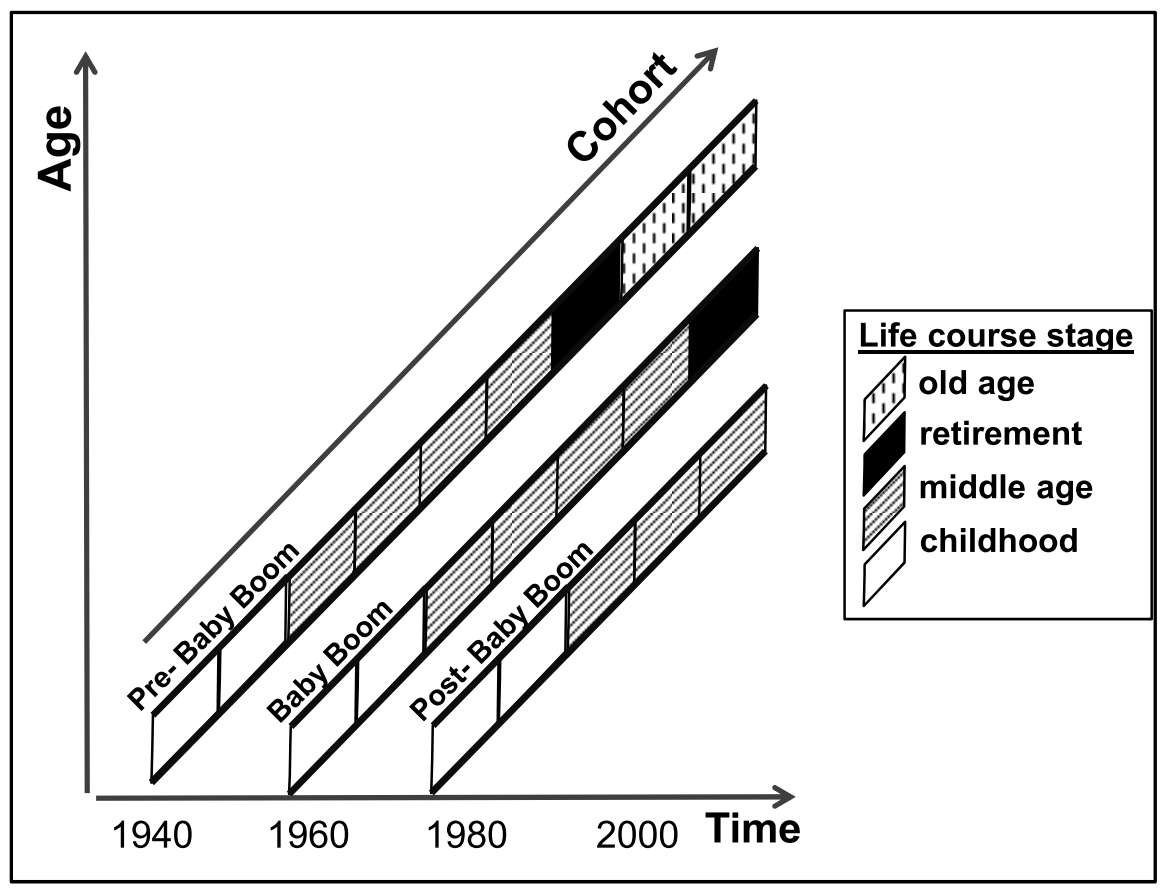

Figure 6 The relation between age and period (time) for three cohorts in a lexis diagram

APC models can be used to disentangle the effects of age, period and cohort, and to determine how the boomers migration behaviour differs from other cohorts. However, disentangling these effects is conceptually and methodologically challenging. On the one hand, the relative importance of individual characteristics (i.e. age) has to be separated from structural factors (i.e. labour market). Conversely, individual and structural factors interact (i.e. age at entry into the labour market) so that the effects of structural factors differ between individuals (Blossfeld, 1986).

In seeking to separate these dimensions, APC models represent each of the effects of age, period and cohort by a set of parameters that describe the propensity to move for each age group, in each period and for each cohort for which data are available. By plotting each set of parameters, the intensity of migration can be compared (a) across age groups, (b) across periods, and (c) across cohorts, and the relativity of these effects can be determined. It can then be established how distinctive the migration behaviour among the baby boomers was and whether they migrated more or less than earlier and later cohorts.

A negative binomial regression model that accounts for overdispersion in the count data was used to disentangle the effects of age, period and cohort on migration among the baby boomers. The model included a dummy variable for each age group, period and cohort. Each of the coefficients of the dummy variables indicates deviation from the migration intensity among the youngest age group (five to nine), the first period (1976-81) and the oldest cohort (1901-06), respectively.

The model parameters for age, period and cohort, were estimated for a series of full, partial and extended models. First, parameters for the full model with age, period and 
cohort (A-P-C) were computed and migration intensity was compared across individual age groups, periods and cohorts in the whole population. Second, partial models were calculated that include all possible combinations of age, period and cohort (A-P, A-C, P-C, $\mathrm{A}, \mathrm{P}, \mathrm{C})$ to compare model fit of the partial models to the full model fit. Third, the full model was extended to determine the effects of cohort size (A-P-C-CS) and economic indicators (A-P-C-EI) on migration. Separate models were run for each of the five types of movements.

The full A-P-C model was estimated with migration probabilities at age $a$, in period $p$, and for birth cohort $c$. This full model served as the baseline against which the fit of all subsequent (partial) APC models was compared. All statistical computations were performed using statistical software (STATA Version 10).

The negative binomial model with a log link takes the following form:

$$
\log \lambda_{a, p, c}=\beta_{0}+\sum_{a=5-9}^{n=75+} \beta_{a} X_{a}+\sum_{p=1976-81}^{n=1996-01} \beta_{p} X_{p}+\sum_{c=1901-06}^{n=1991-96} \beta_{c} X_{c}+\alpha_{a, p, c}+\log \left(t_{a, p, c}\right)
$$

where $\lambda_{a, p, c}$ is the predicted value of migrant count $y_{a, p, c}$ for a particular age group, period and cohort; $x_{a}, x_{p}$ and $x_{c}$ are the independent variables of age group, period and cohort with corresponding regression coefficients $\beta_{n}$. The variable $x_{a}$ (age) is represented by 15 dummies (5-9 to 75+), $x_{p}$ is represented by five period dummies (1976-81 to 1996$01)$ and $x_{c}$ is represented by 19 dummies for cohort (1901-06 to 1991-96). The youngest age group (five to nine), the first period (1976-81) and the oldest cohort (1901-06) were treated as the reference categories and are thus omitted. $\alpha_{a, p, c}$ is the dispersion parameter, and the offset term $t_{a, p, c}$ is the count of people at risk of migrating for a particular age, period and cohort.

Partial models were calculated to compare their model fit to the full model fit. As discussed earlier, the identification problem that arises from the linear dependency between age, period and cohort can be resolved by using partial models. The model fit of the partial models with each possible combination of age, period and cohort was compared to the full model fit to determine if the full model fit was better than the fit of any of the partial models. That is, to determine the value of taking the extra step of disentangling age, period and cohort effects.

In the final step, the full A-P-C model was extended to evaluate the effect of cohort size on migration in the A-P-C-CS (Age-Period-Cohort-Cohort Size) model, and determine the influence of economic indicators on migration in the A-P-C-EI (Age-Period-CohortEconomic Indicators) model. All extended models were run separately for each type of move.

The question of whether the propensity to move was depressed for large cohorts was determined by extending the full A-P-C model to include a dummy representing all cohorts with medium birth cohort size and a dummy for cohorts with large size, while small birth cohorts were the reference category (A-P-C-CS model). Absolute birth cohort 
size was categorised into small, medium and large size cohorts. The migration literature has shown that economic conditions affected the migration behaviour of the US baby boom (Milne, 1993; Nelson and Sewall, 2003; Pandit, 1997a; Plane, 1994). In the present study, the effects of GDP, housing interest rates and unemployment levels on migration were examined by extending the full A-P-C model to include period characteristics (i.e. GDP, housing interest rate and unemployment rate).

\section{Results}

\subsection{Goodness-of-Fit of the APC Models}

The goodness of fit of the full and partial APC models was compared using the Akaike Information Criterion (AIC). The AIC values show that for total mobility the full negative binomial A-P-C model represents the migrant counts most accurately and thus has the best fit (see Table 1). This finding is remarkably consistent across types of moves. Among partial models, the model with dummies for age has the best fit, followed by the model with cohort dummies.

Table 1 AIC of full and partial negative binomial APC models, by type of move

\begin{tabular}{llrrrrrr}
\hline & model & total mobility & same TSD & $\begin{array}{c}\text { metro to } \\
\text { non-metro }\end{array}$ & $\begin{array}{c}\text { non-metro } \\
\text { to metro }\end{array}$ & $\begin{array}{c}\text { non-metro to } \\
\text { non-metro }\end{array}$ & interstate \\
\hline full & A-P-C & 3,266 & 3,169 & 2,278 & 2,126 & 2,074 & 2,642 \\
\hline \multirow{4}{*}{ partial } & A-C & 3,270 & 3,174 & 2,423 & 2,178 & 2,122 & 2,653 \\
& P-C & 3,480 & 3,378 & 2,718 & 2,678 & 2,544 & 2,897 \\
& A & 3,272 & 3,197 & 2,485 & 2,312 & 2,228 & 2,647 \\
& P & 3,665 & 3,560 & 2,785 & 2,863 & 2,813 & 3,086 \\
& C & 3,485 & 3,381 & 2,735 & 2,701 & 2,593 & 2,913 \\
\hline
\end{tabular}

\subsection{Age, Period and Cohort Effects Estimated by the Full APC Model}

The results from the full A-P-C model show a large variation across age groups and cohorts and thus emphasises the importance of disentangling the effects of age from those of cohort. Conversely, period effects play a less important role in the understanding of the determinants of change over time in migration intensities. Figure 7 shows a plot of the incidence rate ratios (IRRs) for age, period and cohort from the full A-P-C model for total mobility with 95 per cent confidence intervals. The IRRs are interpreted as the ratio of the incidence of migration in persons of a particular age, period or cohort to that in the comparison group (i.e. the first age group, period and cohort). Ratios above one indicate higher movement propensities compared to the reference group, while values below one indicate lower risks of moving. 


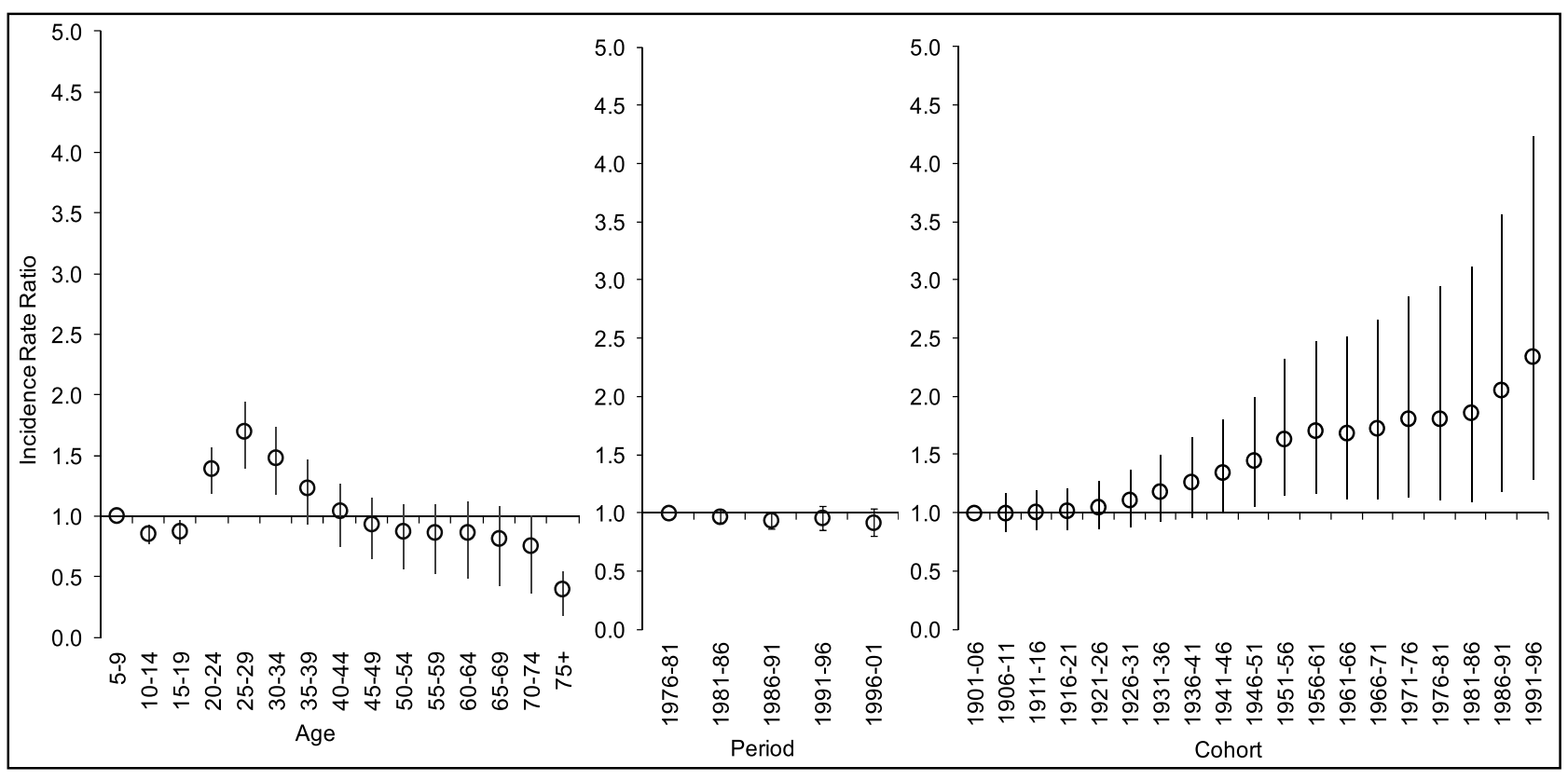

Figure 7 Incidence rate ratios for age, period and cohort estimated for the full A-P-C model (with 95 per cent CIs) for total mobility, persons

It is apparent that the parameters for age closely resemble the pattern found in national migration age profiles, indicating that age is well controlled for in the model. That is, even when period and cohort effects are controlled for, young adults have the highest risk of moving. In contrast, period effects were remarkably stable across time, showing only a slight downward trend. This suggests that period effects, such as economic growth or unemployment rates had a small impact on migration.

The results for cohort clearly show a positive effect. There was a steady but uneven increase in migration propensities across cohorts. The baby boom cohorts born between 1946 and 1976 had a higher risk of migrating than older cohorts, but the post-baby boomer generation is characterised by even higher risks. Cohort effects on migration are thus not restricted to the baby boom but show a continuous upwards trend across cohorts. The relative risk of moving by cohort began to rise in the 1921-26 cohort and rose steadily until the 1956-61 cohort. The migration propensity levelled off for the 1961-66 and 1966-71 cohorts, but resumed its increase among those born between 1966 and 1976, and, after a small dip in the 1976-81 cohort, rose more strongly among the later cohorts. The sharp increase in migration propensity for the latest cohorts may be due to the small number of observations since data for the 1991-96 cohort were available only for the first period 1976-81 (for two APC spaces).

The much lower increase in migration among the younger baby boom cohorts born between 1961 and 1971 is consistent with the findings reported by Pandit (1997a) and Plane and Rogerson (1991), who found that the later US baby boomers moved at reduced rates. However, the general upwards trend stands in sharp contrast to the decline in migration intensity across successive cohorts (at ages 20-24) observed in the US between 
1970 and 1980. The size and timing of the baby boom may play a crucial role in explaining why the direction of change in migration rates across cohorts differed between the two countries. The smaller demographic bulge created by the Australian baby boomers may have resulted in much less competition upon entry into the labour and housing markets and, hence, no lowering of migration levels among the baby boom cohorts.

Relative cohort size may in part account for the levelling off in the increase in total mobility for the 1961-66 cohort. This plateau effect shows some overlap with the findings reported in the North American literature. Pandit (1997a) and Plane and Rogerson (1991) argued that the lowered migration rates among the younger baby boomers in the US were caused by the interacting effects of relative birth cohort size and tough competition at entry into the housing and labour markets. In Australia, the members of the 1961-66 cohort reached young adulthood in the 1980s and entered the housing market in the late 1980s, when interest rates soared. The proportion of 15-24 year olds in the Australian population was relatively high at around 17 per cent during that time. Hence, it appears that the levelling off in the increase of total mobility may have been caused by interactions between (a) the large relative size of the 1961-66 cohort at ages 15-24, and (b) the housing interest rate peak in 1989.

\subsection{Age, Period and Cohort Effects Estimated by the Partial Models}

Further insights into the effects of age, period and birth cohort on migration are obtained by comparing the estimated parameters from the full A-P-C model with those from the partial models, since partial models do not control for all three effects simultaneously. Figure 8 shows plots of the estimated parameters for age and period from the partial model for total mobility. The results for age and period are similar to the overall trend observed for the full A-P-C model, and the differences in parameter estimates between the partial models are small. That is, even if period or cohort effects are not controlled for, the effect for age closely resembles the national migration age profile. Similarly, the effect for period is small and stable across periods, even if age and cohort effects are not controlled for. 


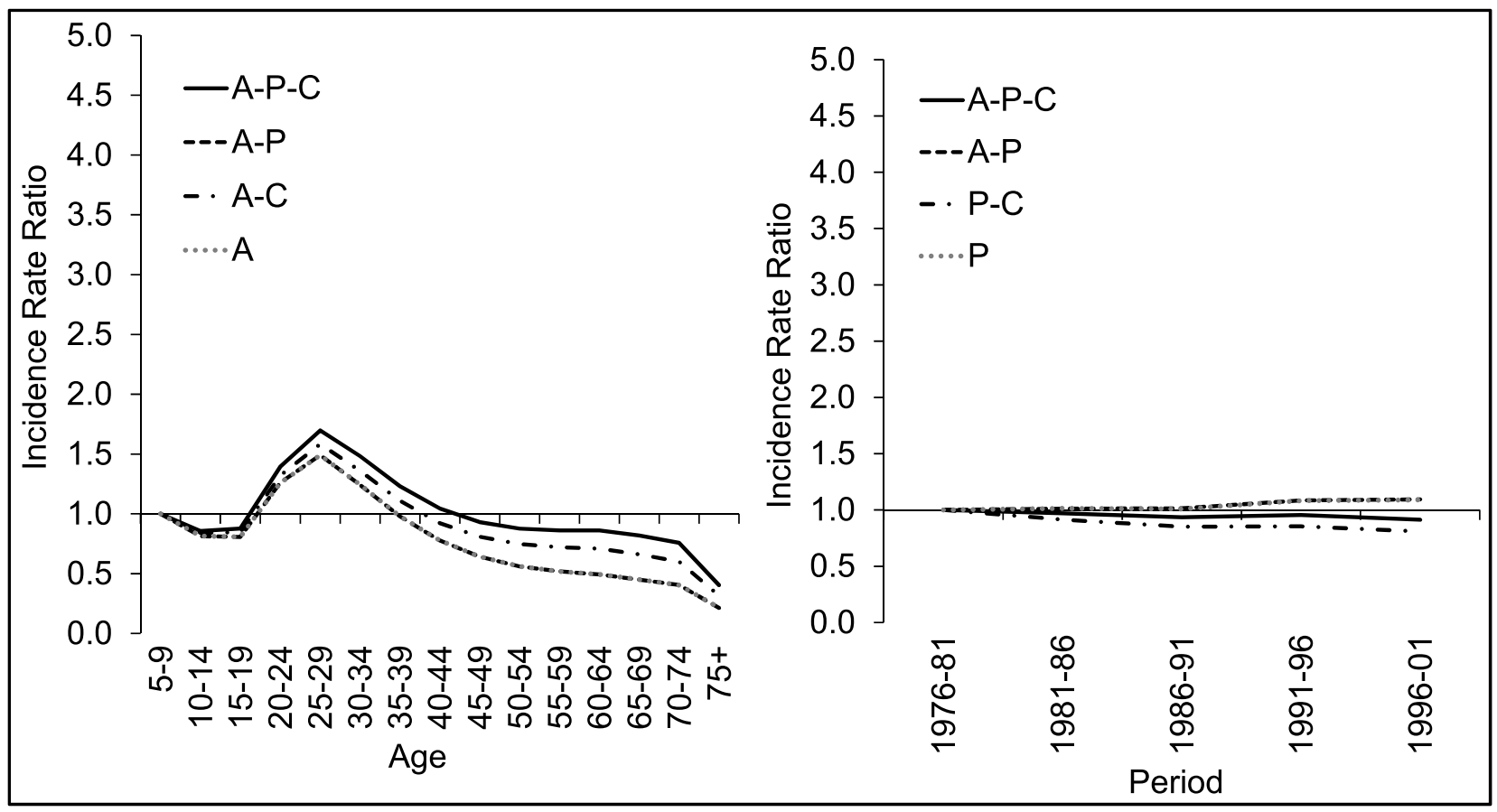

Figure 8 Incidence rate ratios for age (left) and period (right) estimated for the partial models

Figure 9 shows that the results for cohort estimated for the partial models diverge from the overall trend observed for the full A-P-C model. The differences in IRRs for individual cohorts between the partial and the full model highlight the importance of controlling for age in the analysis of cohort effects. The results for the P-C and the $\mathrm{C}$ model show that the cohort effects estimated with these models are much larger than for the A-P-C and the A-C models. This is because cohort effects are confounded with age effects if age is not controlled for in the model.

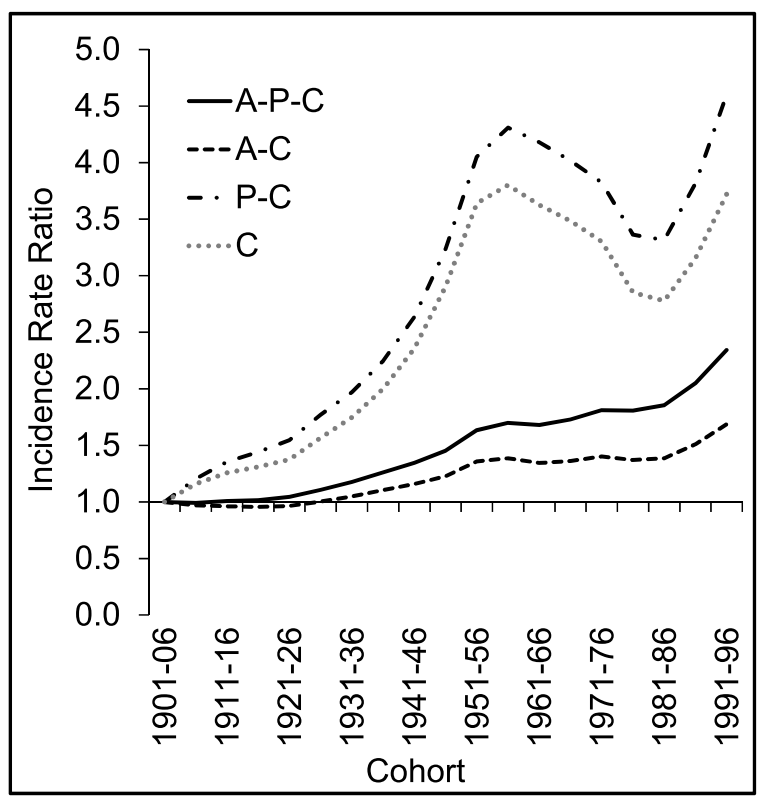

Figure 9 Cohort effects estimated for the partial A-C, P-C and C models for total mobility 


\subsection{The Effects of Cohort Size and Economic Indicators}

A key question that emerges from the comparison of Australian and US findings concerns the relationship between cohort size and the intensity of migration. While the US literature points to lower intensities among members of large cohorts, the results on cohort effects for total mobility suggest that the impact of large cohort size is less pronounced in Australia. Table 2 sets out the IRRs for the full APC model, the extended A-P-C-CS model with dummies for medium and large cohort size, and the extended A-P-C-EI model with economic indicators. The results for the A-P-C-CS model show that medium-sized cohorts (796,001 to 1,360,000 live births over a five-year period) had a 70 per cent higher likelihood of moving than small birth cohorts $(621,000$ to 796,000 live births). Large-sized cohorts $(1,360,001$ to $1,550,000$ live births) were even more likely to move than mediumsized cohorts. The finding that large cohort size appears to have had a positive effect on migration contradicts the US literature (Pandit, 1997a; Plane and Rogerson, 1991).

Turning to the effects of economic indicators, the question arises as to whether cohort effects have been more influential in shaping migration behaviour than economic conditions or housing cost. The results for the A-P-C-EI model with economic indicators show that period effects had negligible influence on migration over the period 1976-81 to 1996-2001. These findings somewhat contradict the US literature, which reports that unemployment rates are negatively related with the intensity of migration. However, the finding that economic indicators are less influential relative to cohort size is in line with the US study by Pandit (1997a) who found that cohort size effects were more influential on US migration rates than economic cycles. 
Table 2 Incidence rate ratios for age, period, cohort, cohort size and economic indicators estimated for the full A-P-C model and extended models, for total mobility, persons

\begin{tabular}{|c|c|c|c|}
\hline Variable & $\begin{array}{l}\text { A-P-C } \\
\text { IRR } \\
\end{array}$ & $\begin{array}{l}\text { A-P-CS } \\
\text { IRR }\end{array}$ & $\begin{array}{l}\text { A-EI-C } \\
\text { IRR }\end{array}$ \\
\hline \multicolumn{4}{|l|}{ AGE } \\
\hline $5-9$ & 1 & 1 & 1.00 \\
\hline $10-14$ & $0.86^{* *}$ & $0.86^{* *}$ & $0.86^{* *}$ \\
\hline $15-19$ & $0.88^{*}$ & $0.88^{*}$ & $0.88^{*}$ \\
\hline $20-24$ & $1.39 * *$ & $1.39 * *$ & $1.39^{* *}$ \\
\hline $25-29$ & $1.70^{* *}$ & $1.70^{* *}$ & $1.69^{* *}$ \\
\hline $30-34$ & $1.48^{* *}$ & $1.48^{* *}$ & $1.48^{* *}$ \\
\hline $35-39$ & $1.23 *$ & $1.23 *$ & 1.23 \\
\hline $40-44$ & 1.04 & 1.04 & 1.04 \\
\hline $45-49$ & 0.93 & 0.93 & 0.92 \\
\hline $50-54$ & 0.88 & 0.88 & 0.87 \\
\hline $55-59$ & 0.86 & 0.86 & 0.85 \\
\hline $60-64$ & 0.86 & 0.86 & 0.85 \\
\hline $65-69$ & 0.82 & 0.82 & 0.81 \\
\hline $70-74$ & 0.76 & 0.76 & 0.75 \\
\hline $75+$ & $0.40^{* *}$ & $0.40 * *$ & $0.40^{* *}$ \\
\hline \multicolumn{4}{|l|}{ PERIOD } \\
\hline $1976-81$ & 1 & 1 & \\
\hline $1981-86$ & 0.97 & 0.97 & \\
\hline 1986-91 & 0.93 & 0.93 & \\
\hline $1991-96$ & 0.95 & 0.95 & \\
\hline 1996-01 & 0.91 & 0.91 & \\
\hline \multicolumn{4}{|l|}{ COHORT } \\
\hline $1901-06$ & 1 & 1 & 1.00 \\
\hline 1906-11 & 0.99 & 0.99 & 0.98 \\
\hline $1911-16$ & 1.01 & 1.01 & 1.00 \\
\hline $1916-21$ & 1.01 & 1.01 & 1.01 \\
\hline $1921-26$ & 1.05 & $0.62 * *$ & 1.04 \\
\hline $1926-31$ & 1.11 & 1.11 & 1.10 \\
\hline $1931-36$ & 1.18 & 1.18 & 1.17 \\
\hline $1936-41$ & $1.26^{*}$ & 1.26 & 1.25 \\
\hline $1941-46$ & $1.35^{*}$ & $0.79 * *$ & $1.33^{*}$ \\
\hline $1946-51$ & $1.45^{* *}$ & $0.85^{* *}$ & $1.44^{*}$ \\
\hline $1951-56$ & $1.63 * *$ & 0.96 & $1.61^{* *}$ \\
\hline $1956-61$ & $1.70^{*}$ & & $1.68^{* *}$ \\
\hline $1961-66$ & $1.68 *$ & $0.72 *$ & $1.66^{*}$ \\
\hline $1966-71$ & $1.73^{*}$ & $0.74^{*}$ & $1.70^{*}$ \\
\hline $1971-76$ & $1.81^{*}$ & $0.77 *$ & $1.78^{*}$ \\
\hline $1976-81$ & $1.81 *$ & $0.77 *$ & $1.78^{*}$ \\
\hline $1981-86$ & $1.85^{*}$ & $0.79 *$ & $1.83^{*}$ \\
\hline 1986-91 & $2.05^{* *}$ & 0.88 & $2.01 *$ \\
\hline $1991-96$ & 2.34 & & $2.29^{* *}$ \\
\hline \multicolumn{4}{|c|}{ COHORT CHARACTERISTICS } \\
\hline small cohort size & & 1.00 & \\
\hline medium cohort size & & $1.70^{* *}$ & \\
\hline large cohort size & & $2.34 * *$ & \\
\hline \multicolumn{4}{|c|}{ PERIOD CHARACTERISTICS } \\
\hline GDP per capita & & & 1.00 \\
\hline Housing interest rate & & & 0.99 \\
\hline Unemployment rate & & & 1.01 \\
\hline Alpha & $0.009^{* *}$ & $0.009^{* *}$ & $0.009^{* *}$ \\
\hline Likelihood ratio chi-square & $460 * *(36 \mathrm{df})$ & $461^{* *}(36 \mathrm{df})$ & $460^{* *}(35 \mathrm{df})$ \\
\hline AIC & 3,266 & 3,269 & 3,268 \\
\hline
\end{tabular}




\subsection{Age, Period and Cohort Effects by Type of Move}

The final question addressed in this paper concerns the differences in cohort effects by type of move. In Australia, the overall trend in cohort-specific mobility is clearly upwards for moves within the same TSD and interstate moves, providing little evidence for differences in the effect of cohort membership on migration by type of move (see Figure 10). Indeed, the results show that cohort effects differ not by distance but by type of movement. Moves within the same TSD and interstate mobility increased across cohorts, while the propensity to move intrastate to non-metropolitan TSDs rose across the cohorts born early in the $20^{\text {th }}$ century, but declined across the cohorts of 1960 to 1990. Each of these three types of movements is discussed in turn.

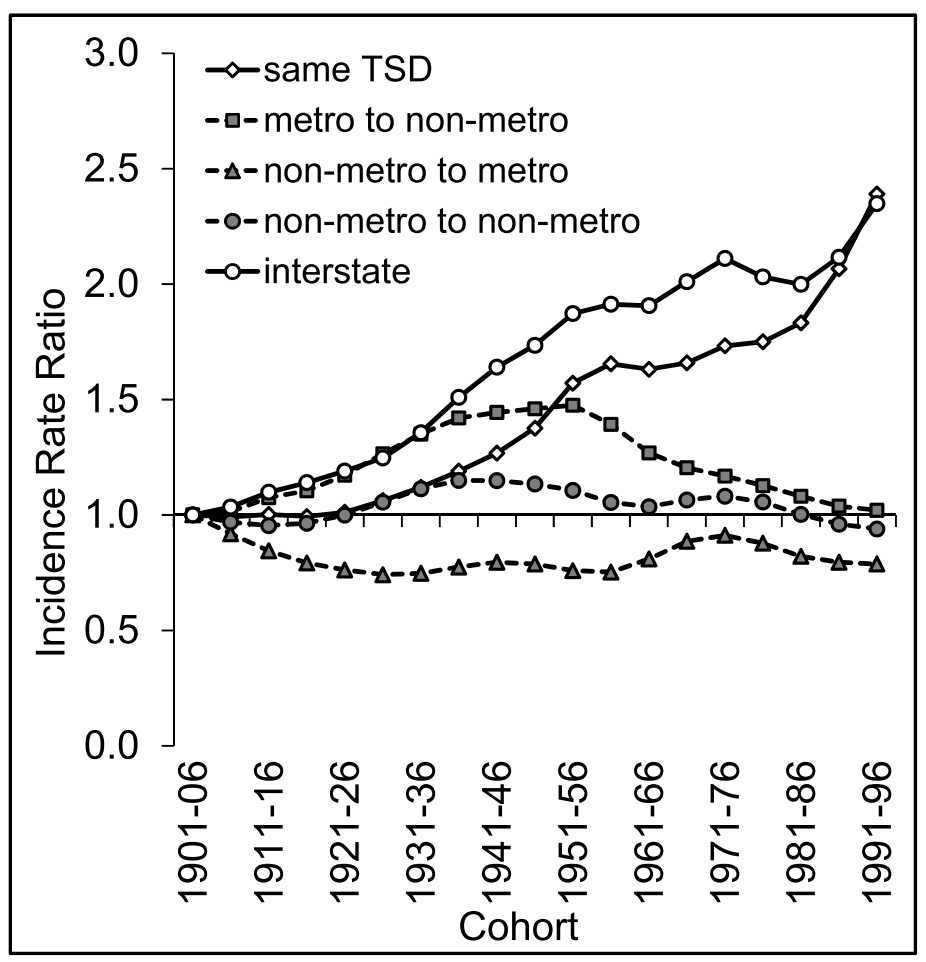

Figure 10 Cohort effects estimated for the full A-P-C model by type of move

The propensity to move within the same TSD increased across cohorts, with a slight plateau for cohorts born in 1961-66. The parameters estimated are similar to those for total mobility discussed earlier, which indicates that it is the pattern for moves within the same TSD that shapes the results observed for total mobility. The increase in movements within the same TSD across cohorts may perhaps be explained in part by reference to the Australian housing market, which is characterised by a large share of owner-occupied dwellings and by an increase in the median age at first home ownership (ABS, 2003; Berry, 1999). Relatively high owner occupancy rates of approximately 70 per cent may have been at work in shaping mobility within the same TSD over the period 1976-81 to 1996-2001. This type of mobility occurs predominantly between suburban areas within Australia's five largest metropolitan centres and is mostly motivated by housing considerations (Hugo et al., 2005). Median age at first home ownership increased 
from 27 years in the early 1980s to 32 years in the early 2000s (Rodrigues, 2003), resulting in young adults remaining longer in rental housing after leaving the parental home around the ages of 17 to 21 . It has been well documented in the literature that those who enter into home ownership are much less likely to move than renters (Bell and Hugo, 2000; Courgeau, 1985; Helderman et al., 2004; Wagner, 1989). Consequently, the intensity of migration within the same TSD rises if first home ownership occurs at a later point in the life-course and the proportion of renters among young adults increases. Changes in housing careers and the timing of life course events may go some way to explaining the increase in the propensity to move within the same TSD across successive cohorts.

The findings show that unlike in the US (Pandit, 1997a) interstate migration has not been increasingly depressed by large cohort size and high unemployment levels. In fact, as argued by Jarvie (Jarvie, 1989:47), the rise in interstate mobility may be population-led rather than employment-led, which means that it is personal attributes and preferences that yield changes in mobility behaviour rather than spatial shifts in labour demand. The rise in interstate mobility has almost certainly been facilitated by the radical transformation of the Australian space economy over the last half century, characterised by the rapid growth of the tourism and service industries not only in the capital cities but also in rural areas (Argent et al., 2008; Collits and Gastin, 1997), and the steady reduction in manufacturing and industrial employment in the rustbelt of south-eastern Australia.

Variations from the overall pattern of increasing migration propensities across cohorts are apparent for intrastate moves. Most important in the context of retirement migration are movements from metropolitan to non-metropolitan region, which tend to be dominated by families with young children and retirees, who move due to lifestyle- or housing-related factors (Burnley and Murphy, 2004). In contrast to moves within the same TSD and interstate mobility, the propensity to move to non-metropolitan regions was highest for the older baby boomers, but showed a subsequent decline across cohort born between 1960 and 1990. It appears that the older boomers were more likely to move from metropolitan to non-metropolitan TSDs than the younger boomers. The youngest pre-baby boomers and the older baby boomers were in the family formation stage in the late 1980s, at the time of the interest rate peak. Hence, there may be three factors that account for the peak in moves away from the capital cities for the baby boomers: (a) the increase in lifestyle-driven migration; (b) urban sprawl and overspill beyond the capital city boundaries; and (c) the interest rate peak in 1989. House prices in the capital cities that became even less affordable when interest rates peaked in 1989, may also have played a role in the flight of baby boomers from the capital cities. This factor would have had a strong influence on the decision of families with young children to move from the inner cities to the urban fringes and non-metropolitan centres along the coast, which are characterised by lower housing costs. Therefore, movement from the capitals to nonmetropolitan TSDs may have been a means of improving lifestyles and avoiding high housing costs.

Turning to differences in the effects of age and period by type of move, the results confirm substantial variation in the effects of age and, to a lesser degree, period by type of move. Figure 11 plots the effects of age and period estimated by the full APC model by type of move. The effects of age closely resemble the general age profile of migration 
(Rogers, 1988), with high migration probabilities among young adults that declined with age. Noteworthy is the retirement peak for 50 to 70 year olds in the age profile of flows from metropolitan to non-metropolitan TSDs. The effects of period were negligible in most periods, but in the 1986-91 interval, the propensity to move from metropolitan to nonmetropolitan TSDs was elevated, while movements from non-metropolitan to metropolitan TSDs depressed. These findings suggest that high interest rates and low housing affordability resulted in increasing outflows from the major metropolitan centres to the urban fringes and smaller coastal towns.

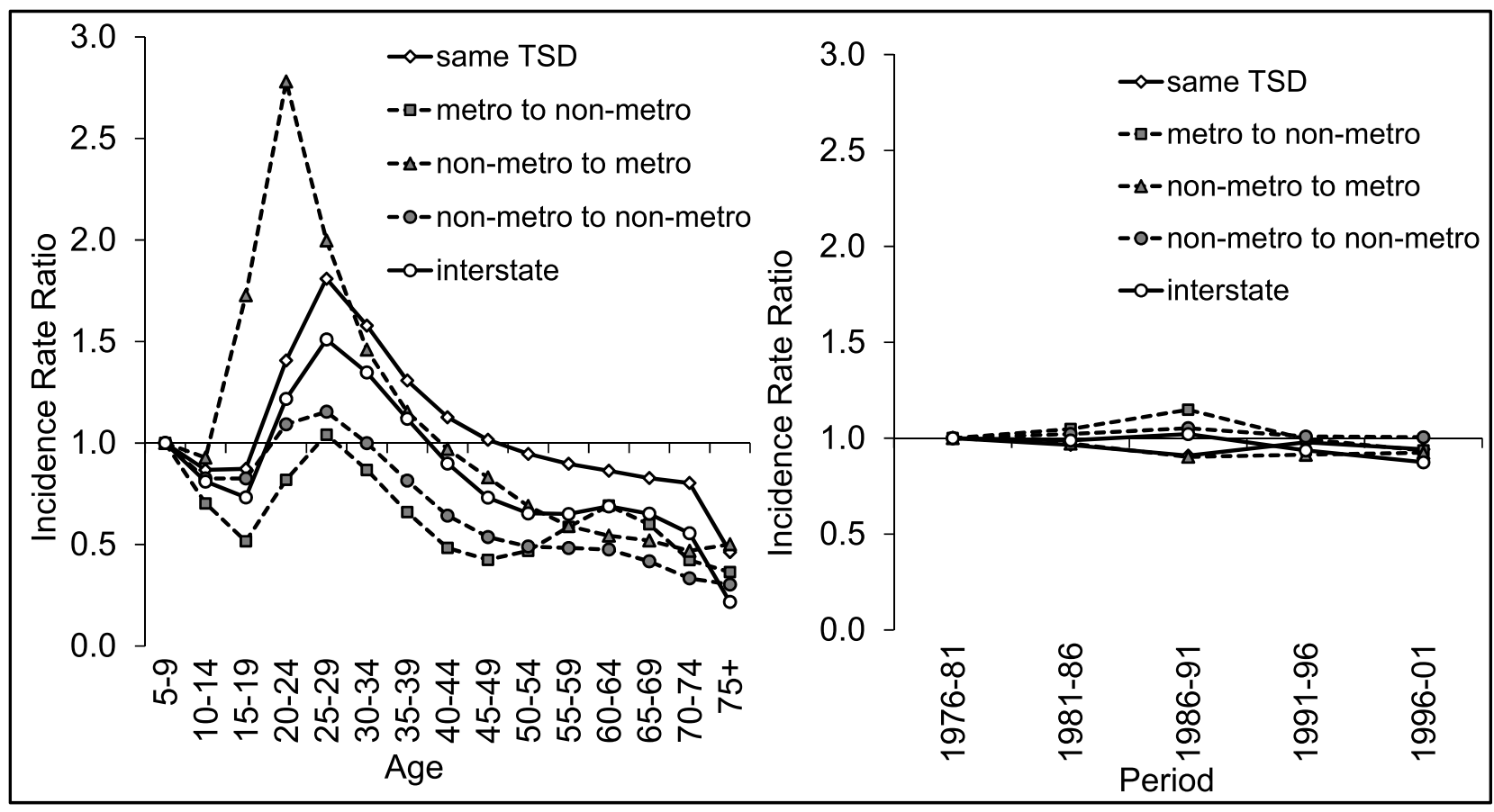

Figure 11 Age and period effects estimated for the full A-P-C model by type of move

While the US literature reports that cohort size has a stronger impact on the propensities to move over longer distances than on short-distance moves (Pandit, 1997a), the results reveal similar cohort effects on the intensity of interstate migration and moves within the same TSD. Specifically, large cohorts were more than twice as likely to move within the same TSD or interstate as small cohorts were (see Figure 12). The results for intrastate moves show no upwards trend, and the effect of cohort size varies by direction of the move. The propensity to move from metropolitan to non-metropolitan TSDs was higher for medium size cohorts than for small and large cohorts, whereas movements in the opposite direction were higher for small cohorts. The effect of cohort size on moves between non-metropolitan TSDs was negligible. The findings suggest that cohort size plays a role in shaping the propensity to move within the same TSD and interstate, while intrastate moves appear to be influenced by other demographic or economic factors. 


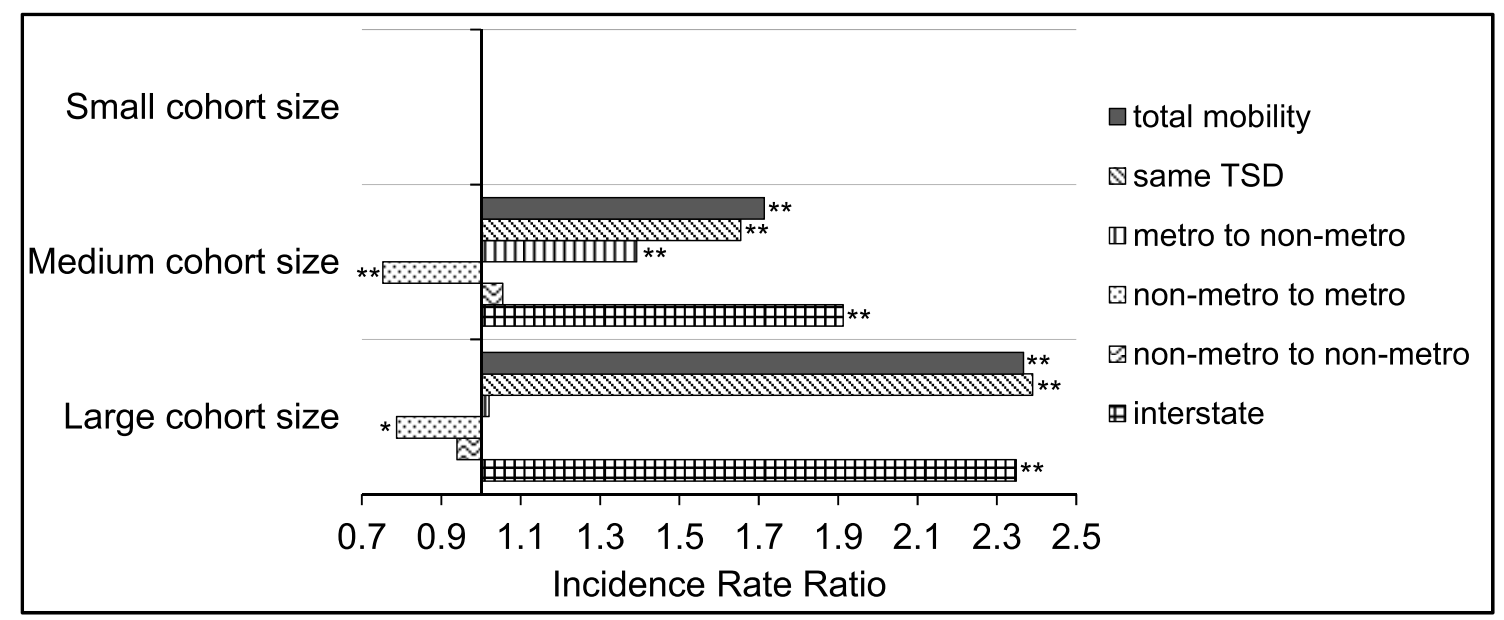

Figure 12Cohort size effects estimated for the A-P-C-CS model by type of move $(* \mathrm{p} \leq .05 ; * * \mathrm{p} \leq .01)$

Turning to the effects of economic indicators by type of move, the result from the A-P-C-EI Model shows that GDP, housing interest rates and unemployment rates influenced the propensity to move intrastate, while the effects on total mobility were negligible (see Figure 13). Specifically, high housing interest rates had a positive effect on moves to non-metropolitan TSDs and interstate mobility, whereas the effect on the propensity to move within the same TSD and from non-metropolitan to metropolitan TSDs was negative. This finding suggests that movement to non-metropolitan regions may have been a means of avoiding or escaping high housing costs in the capital cities.

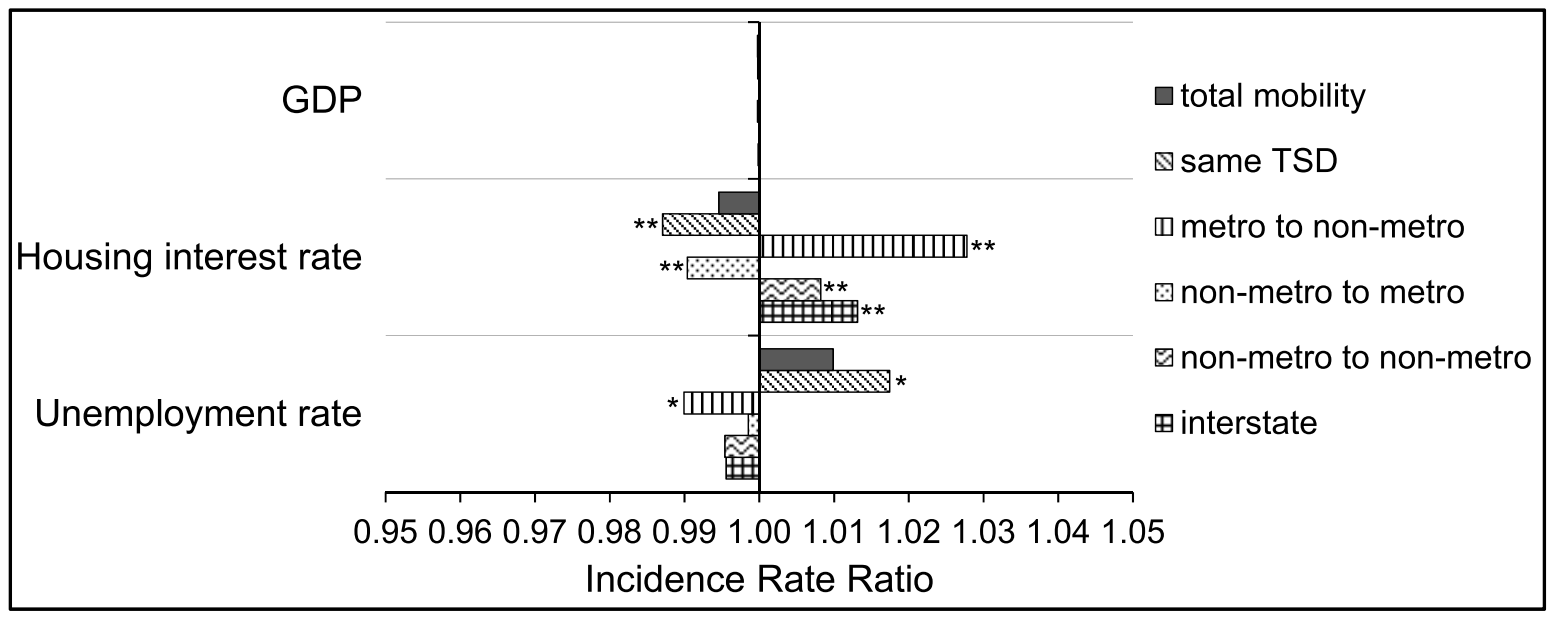

Figure 13 Period effects estimated for the A-P-C-EI model by type of move $(* \mathrm{p} \leq .05 ; * * \mathrm{p} \leq .01)$

The effect of unemployment differs from that of cohort size: High unemployment encouraged moves within the same TSD, while at the same time decreasing the propensity to move over longer distances, especially from metropolitan to non-metropolitan TSDs. In comparison to the findings reported for the US (Milne, 1993; Pandit, 1997a), economic 
factors appear to play a much smaller role in shaping migration intensities in the Australian context.

\section{Conclusion}

The analysis presented here found no clear evidence of a negative effect of large cohort size on migration. A series of APC models based on the negative binomial distribution were used to disentangle the effects of age, period and cohort on migration intensity. To avoid the identifiability problem common to APC models, the period-cohort spaces were split into 'younger' and 'older' APC spaces (Bell and Rees, 2006). The results demonstrated that the relative contribution of age to change in migration intensity is largest in relative terms, followed by cohort, while the effects of period were much more subtle.

While the behaviour of the Australian baby boomers in general has been claimed to be as distinctive as that of their North American counterparts (Productivity Commission, 2008), the negative effect of birth cohort size on migration does not appear to hold in the Australian context. Moreover, the results demonstrated that cohort effects differ by type of move. Local and interstate migration increased strongly across successive cohorts, although the increase levelled off slightly for the baby boomer cohort born in 1961-66. Conversely, the rate of counter-urban movement peaked during the baby boomer cohorts and declined for later cohorts. This divergence from the US-based evidence regarding cohort effects is largely due to the different timing and magnitude of the Australian baby boom compared to the US. The baby boom in Australia was delayed and much smaller in terms of absolute and relative cohort size than in the US, which means that the demographic bulge created by the baby boom was much smaller. While the intensity of migration among the Australian baby boomers was higher compared to earlier cohorts, their migration behaviour appears to have been shaped by factors other than cohort size. Given the importance of lifestyle- and housing-related factors in shaping migration behaviour in Australia, preferences for specific destination types and housing market dynamics seem to be more important predictors of migration than cohort size. 


\section{References}

ABS. 1998. Australian Social Trends, Family Formation: Family planning. Canberra.

---. 2003. Home Ownership Across Australia' in Australian Social Trends. Canberra.

Argent, N., F. Rolley \& J. Walmsley. 2008. The Sponge City Hypothesis: does it hold water? Australian Geographer 39: 109 - 130.

Bell, M. \& G. Hugo. 2000. Internal migration in Australia 1991-96: Overview and the overseas-born. Canberra: Department of Immigration and Multicultural Affairs.

Bell, M. \& P. Rees. 2006. Comparing migration in Britain and Australia: harmonisation through use of age - time plans. Environment and Planning A 38: 959-988.

Bell, M., P. Rees, M. Blake \& O. Duke-Williams. 1999. An age-period-cohort data base of Inter-regional migration in Australia and Britain, 1976-96. School of Geography, University of Leeds, United Kingdom.

Berry, M. 1999. Unravelling the "Australian Housing Solution": the Post-War Years. Housing, Theory and Society 16: 106 - 123.

Blossfeld, H.-P. 1986. Career opportunities in the Federal Republic of Germany: a dynamic approach to the study of life-course, cohort, and period effects. Eur Sociol Rev 2: 208-225.

Burnley, I. \& P. A. Murphy. 2004. Sea Change: Movement from Metropolitan to Arcadian Australia. Sydney, Australia: University of New South Wales Press.

Collits, P. \& B. Gastin. 1997. Big town small town: the centralisation of services and economic activity, the decline of small towns and the policy response in New South Wales. Regional Policy and Practice 6: 9-22.

Courgeau, D. 1985. Interaction between spatial mobility, family and career life-cycle: A French survey. Eur Sociol Rev 1: 139-162.

Fienberg, S. E. \& W. M. Mason. 1979. Identification and estimation of age-cohort models in the analysis of discrete archival data. In Sociological Methodology, ed. K. F. Schuessler, 1-67. San Francisco: Josey-Bass.

Flatau, P., M. Forbes, P. H. Hendershott \& G. A. Wood. 2003. Homeownership and Unemployment: The Roles of Leverage and Public Housing. SSRN eLibrary.

Frey, W. H. 1986. Lifecourse migration and redistribution of the elderly across US regions and metropolitan areas. Econ Outlook USA 13.

Haas, W. H. \& W. J. Serow. 2002. The Baby Boom, Amenity Retirement Migration, and Retirement Communities: Will the Golden Age of Retirement Continue? Research on Aging 24: 150-164.

Helderman, A. C., C. H. Mulder \& M. Ham. 2004. The changing effect of home ownership on residential mobility in the Netherlands, 1980-98. Housing Studies 19: 601 - 616. 
Hugo, G., K. Harris, E. Bamford \& J. Nottage. 2005. Internal Migration in Australia Analysis of Australia Post Mail Redirection Database. GISCA (The National Centre for Social Applications of GIS), University of Adelaide.

Jarvie, W. K. 1989. Changes in Internal Migration in Australia: Population- or Employment-Led? In Regional Structure and Change: Experiences and Prospects in Two Mature Economies, eds. L. J. Gibson \& R. J. Stimson, 47-60. Peace Dale, Rhode Island: Regional Science Research Institute.

Mason, K. O., W. M. Mason, H. H. Winsborough \& W. K. Poole. 1973. Some Methodological Issues in Cohort Analysis of Archival Data. American Sociological Review 38: 242-258.

Mason, W. \& S. Fienberg. 1985. Cohort Analysis in Social Research: Beyond the Identification Problem. New York: Springer.

Mason, W. M. \& H. L. Smith. 1985. Age-Period-Cohort Analysis and the Study of Deaths from Pulmonary Tuberculosis. In Cohort Analysis in Social Research. New York: Springer.

Milne, W. J. 1993. Macroeconomic Influences on Migration. Regional Studies 27: 365 373.

Nelson, P. B. \& A. Sewall. 2003. Regional Comparisons of Metropolitan and Nonmetropolitan Migration in the 1970s and 1980s: Age and Place Implications. The Professional Geographer 55: 83 - 99.

O'Brien, R. M. 2000. Age Period Cohort Characteristic Models. Social Science Research 29: 123-139.

Pandit, K. 1997a. Cohort and Period Effects in U.S. Migration: How Demographic and Economic Cycles Influence the Migration Schedule. Annals of the Association of American Geographers 87: 439-450.

Pandit, K. 1997b. Demographic cycle effects on migration timing and the delayed mobility phenomenon. Geographical Analysis 29: 187-199.

Plane, D. A. 1992. Age-Composition Change and the Geographical Dynamics of Interregional Migration in the U.S. Annals of the Association of American Geographers 82: 64 - 85.

Plane, D. A. 1994. The wax and wane of interstate migration patterns in the USA in the 1980s: a demographic effectiveness field perspective. Environment and Planning $A$ 26: $1545-1561$.

Plane, D. A. \& P. A. Rogerson. 1991. Tracking the baby boom, the baby bust, and the echo generations: How age composition regulates U.S. migration. The Professional Geographer 43: 416-430.

Productivity Commission. 2008. Trends in aged care services: Some implications In Commission research paper. Canberra: Productivity Commission.

Rees, P., M. Bell, O. Duke-Williams \& M. Blake. 2000. Problems and solutions in the measurement of migration intensities: Australia and Britain compared. Population Studies: A Journal of Demography 54: 207 - 222. 
Robertson, C. \& P. Boyle. 1998. Age-period-cohort analysis of chronic disease rates. I: modelling approach. Statistics in Medicine 17: 1305-1323.

Rodrigues, M. 2003. First home buyers in Australia. Domestic Economy Division, Australian Treasury.

Rogers, A. 1988. Age Patterns of Elderly Migration: An International Comparison. Demography 25: 355-370.

Rogerson, P. A. 1987. Changes in US National Mobility Levels. The Professional Geographer 39: 344 - 351.

Wagner, M. 1989. Räumliche Mobilität im Lebensverlauf. Stuttgart: Enke.

Wilmoth, J. R. 1990. Variation in vital rates by age, period, and cohort. Sociological Methodology 20: 295-336.

Yang, Y. 2007. Age/Period/Cohort Distinctions. In Encyclopaedia of Health and Aging, ed. K. Markides, 20-22. Thousand Oaks, CA: Sage.

Yang, Y., S. Schulhofer-Wohl, W. Fu \& K. Land. 2008. The Intrinsic Estimator for AgePeriod-Cohort Analysis: What It Is and How To Use It. American Journal of Sociology 113. 


\section{VIENNA INSTITUTE OF DEMOGRAPHY}

\section{Working Papers}

Grafeneder-Weissteiner, Theresa, Ingrid Kubin, Klaus Prettner, Alexia Prskawetz, and Stefan Wrzaczek, Coping with Inefficiencies in a New Economic Geography Model, VID Working Paper 04/2012.

Goujon, Anne, Éric Caron Malenfant, and Vegard Skirbekk, Towards a Catholic North America? Projections of Religion in Canada and the US beyond the Mid-21st Century, VID Working Paper 03/2012.

Di Giulio, Paola, Christoph Bühler, Andreas Ette, Romina Fraboni, and Kerstin Ruckdeschel, Social Capital and Fertility Intentions: The Case of Italy, Bulgaria, and West Germany, VID Working Paper 02/2012.

Abel, Guy J., Estimating Global Migration Flow Tables Using Place of Birth Data, VID Working Paper 01/2012.

Ediev, Dalkhat M., At Modal Age at Death, the Hazard Rate is Determined by its Derivative, VID Working Paper 08/2011.

Zeman, Kryštof, Tomáš Sobotka, Richard Gisser, Maria Winkler-Dworak, and Wolfgang Lutz, Geburtenbarometer Vienna: Analysing Fertility Convergence between Vienna and Austria, VID Working Paper 07/2011 (English and German versions available).

Feichtinger Gustav, Michael Kuhn, Alexia Prskawetz, and Stefan Wrzaczek, The Reproductive Value as Part of the Shadow Price of Population, VID Working Paper 06/2011.

Barakat, Bilal, Time is Money: Could Deferred Graduate Retirement Finance Higher Education?, VID Working Paper 05/2011.

Riosmena, Fernando, Maria Winkler-Dworak, Alexia Prskawetz, and Gustav Feichtinger, The Impact of Policies Influencing the Demography of Age Structured Populations: Lessons from Academies of Science, VID Working Paper 04/2011.

Buber, Isabella and Katrin Fliegenschnee, Are you Ready for a Child? A Methodological Triangulation on Fertility Intentions in Austria, VID Working Paper 03/2011.

Fent, Thomas, Belinda Aparicio Diaz, and Alexia Prskawetz, Family Policies in the Context of Low Fertility and Social Structure, VID Working Paper 02/2011.

The Vienna Institute of Demography Working Paper Series receives only limited review. Views or opinions expressed herein are entirely those of the authors. 